\title{
Pre-Test Assessment of the Use Envelope of the Normal Force of a Wind Tunnel Strain-Gage Balance
}

\author{
N. Ulbrich ${ }^{\dagger}$ \\ Jacobs Technology Inc., Moffett Field, California 94035
}

The relationship between the aerodynamic lift force generated by a wind tunnel model, the model weight, and the measured normal force of a straingage balance is investigated to better understand the expected use envelope of the normal force during a wind tunnel test. First, the fundamental relationship between normal force, model weight, lift curve slope, model reference area, dynamic pressure, and angle of attack is derived. Then, based on this fundamental relationship, the use envelope of a balance is examined for four typical wind tunnel test cases. The first case looks at the use envelope of the normal force during the test of a light wind tunnel model at high subsonic Mach numbers. The second case examines the use envelope of the normal force during the test of a heavy wind tunnel model in an atmospheric low-speed facility. The third case reviews the use envelope of the normal force during the test of a floor-mounted semi-span model. The fourth case discusses the normal force characteristics during the test of a rotated full-span model. The wind tunnel model's lift-toweight ratio is introduced as a new parameter that may be used for a quick pre-test assessment of the use envelope of the normal force of a balance. The parameter is derived as a function of the lift coefficient, the dimensionless dynamic pressure, and the dimensionless model weight. Lower and upper bounds of the use envelope of a balance are defined using the model's lift-to-weight ratio. Finally, data from a pressurized wind tunnel is used to illustrate both application and interpretation of the model's lift-to-weight ratio.

\section{Nomenclature}

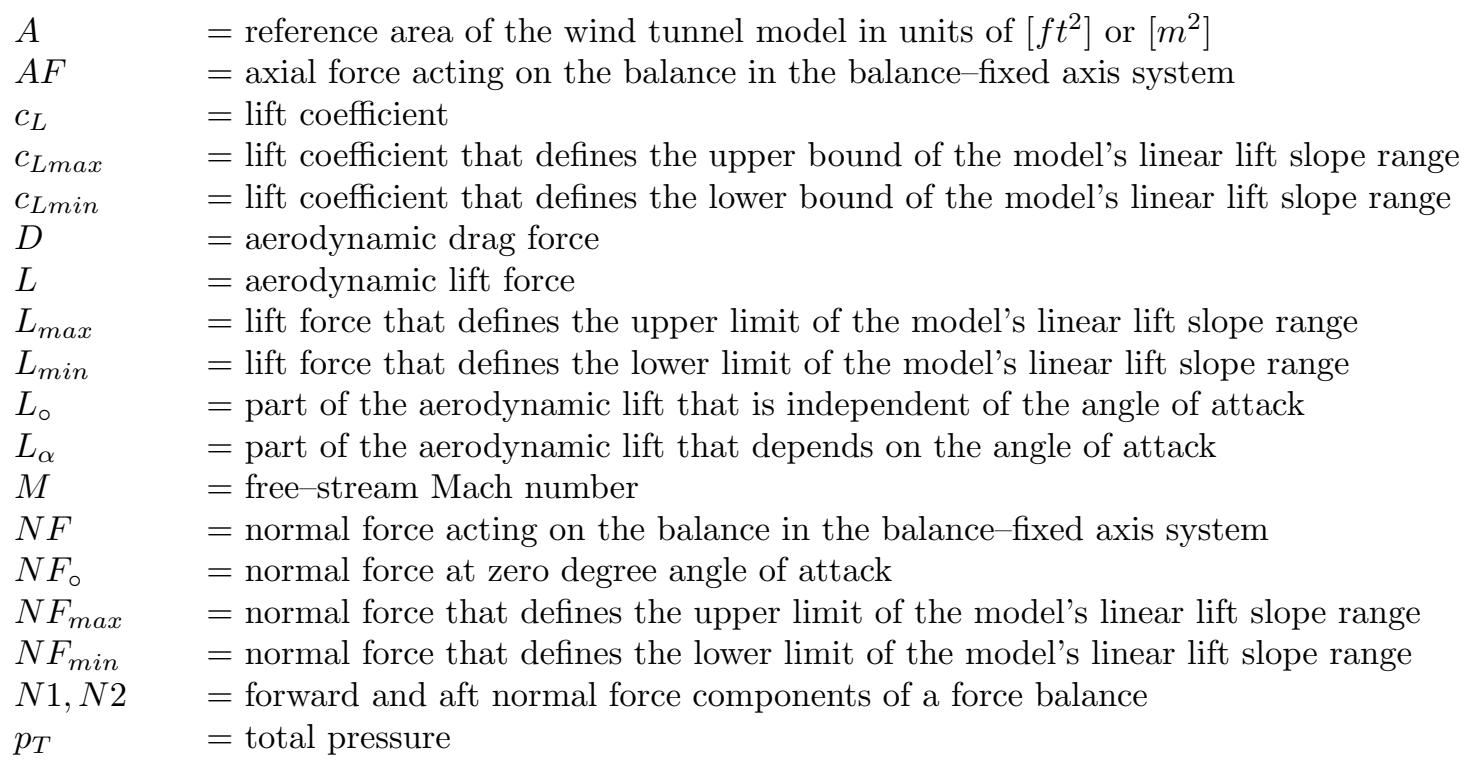

\footnotetext{
$\dagger$ Aerodynamicist, Jacobs Technology Inc.
} 


\begin{tabular}{|c|c|}
\hline$p_{T \circ}$ & $=$ mean sea-level pressure; equals $2116.2\left[\mathrm{lbf} / \mathrm{ft} t^{2}\right]$ or $101325\left[\mathrm{~N} / \mathrm{m}^{2}\right]$ \\
\hline$q$ & $=$ dynamic pressure in units of $\left[l b f / f t^{2}\right]$ or $\left[N / m^{2}\right]$ \\
\hline$R M$ & $=$ rolling moment \\
\hline$r A F$ & $=$ electrical output of the axial force gage of a force balance \\
\hline$r N 1, r N 2$ & $=$ electrical outputs of the forward and aft normal force gages of a force balance \\
\hline$r R M$ & $=$ electrical output of the rolling moment gage of a force balance \\
\hline$r S 1, r S 2$ & $=$ electrical outputs of the forward and aft side force gages of a force balance \\
\hline$S$ & $=$ sensitivity of a balance gage expressed in units of $[\mu V / V]$ per load unit \\
\hline$S 1, S 2$ & $=$ forward and aft side force components of a force balance \\
\hline$W$ & $=$ weight of the wind tunnel model in units of $[l b f]$ or $[N]$ \\
\hline$\alpha$ & $=$ angle of attack of the wind tunnel model \\
\hline$\alpha_{\max }$ & $=$ angle of attack that defines the upper bound of the model's linear lift slope range \\
\hline$\alpha_{\min }$ & $=$ angle of attack that defines the lower bound of the model's linear lift slope range \\
\hline$\gamma$ & $=$ ratio of specific heats of a gas; ratio equals $\approx 1.4$ for air \\
\hline$\delta$ & $=$ dimensionless dynamic pressure \\
\hline$\Delta L$ & $=$ balance dependent force measurement error \\
\hline$\Delta N F$ & $=$ normal force measurement error \\
\hline$\Delta N 1$ & $=$ forward normal force measurement error \\
\hline$\Delta N 2$ & $=$ aft normal force measurement error \\
\hline$\eta, \mu$ & $=$ coordinates that define the wind axis system \\
\hline$\eta^{\prime}, \mu^{\prime}$ & $=$ coordinates that define the balance-fixed axis system \\
\hline$\Lambda$ & $=$ relative error of the lift force expressed in percent \\
\hline & $=$ dimensionless weight of the wind tunnel model \\
\hline
\end{tabular}

\section{Introduction}

A typical wind tunnel customer is interested in the accurate measurement of all aerodynamic forces and moments that act on the test article during a wind tunnel test. Two fundamentally different approaches are used in the aerospace testing community for this purpose. The first approach combines surface pressure measurements on the test article with numerical integration in order to obtain an estimate of the aerodynamic loads. Surface pressure measurements can be obtained either by installing surface pressure ports or by applying pressure sensitive paint to the model surface. These techniques have the advantage that localized load measurements can easily be performed on the model. However, loads associated with skin-friction effects are usually not included in loads that result from the numerical integration of surface pressures. In addition, data acquisition and processing of surface pressure measurements can be a challenging task for a test article with a highly complex geometry.

The second approach uses either an external or internal strain-gage balance for the measurement of aerodynamic loads. In that case, the "raw" balance loads are typically the sum of aerodynamic loads and loads that are caused by the weight of the wind tunnel model. Consequently, the desired aerodynamic loads have to be determined by subtracting loads associated with the weight of the model from the measured "raw" balance loads. Strain-gage balance measurements have the advantage that the integrated total load on the wind tunnel model can easily be measured. However, it is much more difficult to measure localized loads on parts of the model as each localized measurement requires the installation of a dedicated strain-gage balance on the model itself.

Loads associated with the weight of the model can have a significant impact on the actual use envelope of a strain-gage balance during a wind tunnel test. A good qualitative and quantitative understanding of the relationship between aerodynamic loads and loads caused by the weight of the model is critical so that (i) the most suitable balance can be chosen for a specific test and (ii) the impact of the model weight on the accuracy of the measurement of the aerodynamic loads can be assessed with confidence.

In general, the model weight significantly influences the measurement of the normal force in the balancefixed axis system if (i) the model of a traditional airplane is tested such that the generated lift force points towards the ceiling or floor of the wind tunnel, (ii) the model's angle of attack stays within the limits of 
the linear lift slope range, and (iii) the model's roll and yaw angles are close to zero degrees. Therefore, in order to limit the scope of the current paper, the author decided to focus his investigations on a description of the use envelope of the normal force as the normal force of the balance, the weight of the model, and the aerodynamic lift force can easily be related to each other.

First, the basic relationship between normal force, model size, model weight, dynamic pressure, angle of attack, and lift coefficient is derived in the next section of the paper. Then, the expected normal force range of four typical wind tunnel test situations is discussed. In the next step, the lift-to-weight ratio of the wind tunnel model is introduced as a new metric to help quantify the use envelope of the normal force. Finally, numerical examples from tests in the NASA Ames 11-Ft Transonic Wind Tunnel are used to illustrate the application of a wind tunnel model's lift-to-weight ratio to real-world test situations.

\section{Normal Force Description}

The fundamental relationship needs to be derived that connects the measured normal force of the balance to model size, weight, dynamic pressure, angle of attack, and lift force. Figure 1 shows the connection between aerodynamic lift and model weight for a typical wind tunnel model. Figure 2 summarizes all principle forces that act in both the wind and balance-fixed axis system of an unpowered wind tunnel model.

The axis systems used in Fig. 2 were intentionally chosen such that the definition of the positive coordinate direction would match the definition of the positive force direction. This choice differs from the definition commonly used in wind tunnel tests (see Ref. [1], p. 3, or Ref. [2], p. 242, for a discussion of this topic). However, it makes it easier for the reader to follow the derivation of the relationship between the different forces that act on a wind tunnel model. The coordinates $\eta$ and $\mu$ describe forces in the chosen wind axis system. The coordinates $\eta^{\prime}$ and $\mu^{\prime}$ describe forces in the chosen balance-fixed axis system. Then, after projecting loads from the wind axis system to the balance-fixed axis system, we get:

$$
N F=-W \cdot \cos \alpha+L \cdot \cos \alpha+D \cdot \sin \alpha
$$

Now, after assuming that the model is tested within the limits of the moderate angle of attack range of $\pm 10^{\circ}$, we get the following approximations for $\sin \alpha$ and $\cos \alpha$ :

$$
\begin{aligned}
& |\alpha| \leq 10^{\circ} \Longrightarrow|\cos \alpha| \geq 0.98 \ldots \approx 1 \\
& |\alpha| \leq 10^{\circ} \Longrightarrow|\sin \alpha| \leq 0.17 \ldots \approx 0
\end{aligned}
$$

The lift force is typically one order of magnitude greater than the associated drag force for the chosen angle of attack range. Therefore, because $|\cos \alpha| \gg|\sin \alpha|$ and $L \gg D$, it is concluded that

$$
|L \cdot \cos \alpha| \gg|D \cdot \sin \alpha|
$$

The inequality $(2 c)$ given above and the approximation $|\cos \alpha| \approx 1$ given in Eq. (2a) can be used to simplify Eq. (1). Consequently, we get the following approximation of the measured normal force for the moderate angle of attack range of $\pm 10^{\circ}$ :

$$
N F \approx-W+L
$$

It remains to replace the lift $L$ in Eq. (3) by using a reasonable approximation. In principle, the lift $L$ can be decomposed into a part $L_{\circ}$ that is independent of the angle of attack of the wind tunnel model and a part $L_{\alpha}$ that linearly depends on the angle of attack. Then, we can write:

$$
L=L_{\circ}+L_{\alpha}
$$

The part $L_{\alpha}$ can be substituted by using the lift coefficient and the lift curve slope. Consequently, we get:

$$
L_{\alpha}=q \cdot A \cdot c_{L}=q \cdot A \cdot \frac{d c_{L}}{d \alpha} \cdot \alpha
$$

American Institute of Aeronautics and Astronautics 
Finally, assuming that the wind tunnel model has a symmetric airfoil section (i.e., $L_{\circ}=0$ ) and after replacing the term $L_{\alpha}$ of Eq. (4a) by the right-hand side of Eq. (4b), we get the following approximation of the aerodynamic lift of the wind tunnel model:

$$
L \approx q \cdot A \cdot \frac{d c_{L}}{d \alpha} \cdot \alpha
$$

Now, the right-hand side of Eq. (4c) may be used to replace the aerodynamic lift in Eq. (3). Then, the normal force of the balance can be expressed as follows:

$$
N F \approx \underbrace{-W}_{\text {constant }}+\underbrace{\left[q \cdot A \cdot \frac{d c_{L}}{d \alpha}\right]}_{\text {slope }} \cdot \alpha
$$

The model weight $W$, dynamic pressure $q$, reference area $A$, and lift curve slope $d c_{L} / d \alpha$ are constants as long as the model and the dynamic pressure are not changed during a wind tunnel run. Therefore, Eq. (5) describes the normal force of the balance as a linear function of the model's angle of attack assuming that the model stays within the limits of the linear lift slope range. Equation (5) can be summarized as follows:

\section{NORMAL FORCE DURING WIND TUNNEL TEST}

$$
\begin{gathered}
N F(\alpha) \approx N F_{\circ}+\frac{d N F}{d \alpha} \cdot \alpha \\
\text { where } \\
N F_{\circ}=-W \\
\frac{d N F}{d \alpha}=q \cdot A \cdot \frac{d c_{L}}{d \alpha}
\end{gathered}
$$

Equation $(6 a)$ can be used for a systematic discussion of four typical wind tunnel test cases that represent different relationships between the aerodynamic lift force, the model weight, and the use envelope of the normal force of the balance. These four cases are discussed in detail in the next section of the paper.

\section{Discussion of Typical Wind Tunnel Test Situations}

\section{A. General Remarks}

It was shown in the previous section that the behavior of the normal force of a strain-gage balance can be described as a linear function of the angle of attack as long as the model itself stays within the limits of the linear lift slope range. In addition, the model weight causes a shift of this linear function in the direction of the negative part of the normal force axis. Four typical wind tunnel test cases can qualitatively be studied by using these two characteristics of the behavior of the normal force. In all four cases it is assumed that the test article is an unpowered model of an airplane with symmetric airfoil sections. In addition, it is assumed for the first two test cases that the positive lift force points towards the ceiling of the tunnel (see, e.g., Fig. 1). The first case looks at the test of a "light" model at high subsonic Mach numbers. The second case examines characteristics of the test of a "heavy" model at low subsonic Mach numbers. The third case discusses the wind tunnel test of a floor-mounted semi-span model. Finally, the fourth case reviews characteristics of the wind tunnel test of a rotated full-span model. The four test cases are reviewed in detail in the following sections. Afterwards, a conservative estimate of the relative error of the lift force is derived that can be used to assess the impact of the weight on the overall accuracy of the force measurement. 


\section{B. Light Model in Pressurized Wind Tunnel at High Subsonic Mach Number}

The expected behavior of the normal force for a light wind tunnel model tested at high subsonic Mach numbers is investigated in this section. Figure 3a summarizes key characteristics of the expected behavior of the normal force as a function of the angle of attack. The high dynamic pressures needed in order to achieve a high subsonic Mach number cause a large maximum positive lift on the test article that is significantly larger than the weight of the model. This observation can be described by the following inequality:

$$
\frac{\text { maximum positive lift }}{\text { model weight }} \gg 1
$$

This observation also means that the range of the normal force during the wind tunnel test will cover a good percentage of the range of the normal force that may have been applied during the calibration of the balance. In other words, the use envelope of the balance $\left(N F_{\min } \leq N F \leq N F_{\max }\right)$ covers a significant portion of the normal force range that was applied during the balance calibration $\left(-N F_{c a l} \leq N F \leq+N F_{\text {cal }}\right)$.

\section{Heavy Model in Atmospheric Wind Tunnel at Low Subsonic Mach Number}

The expected behavior of the normal force is completely different for a heavy wind tunnel model that is tested in an atmospheric facility at low subsonic Mach numbers. Figure 3b summarizes key characteristics of the behavior of the normal force for this situation. Low dynamic pressures experienced in an atmospheric tunnel at low Mach numbers cause a relatively small maximum positive lift on the test article that is often close to the weight of the model. This observation can be described by the following approximation:

$$
\frac{\text { maximum positive lift }}{\text { model weight }} \approx 1
$$

Consequently, the range of the normal force during the test is a much smaller part of the total range that may have been applied during the calibration of the balance. Most data points of an angle of attack sweep may be recorded at a negative normal force. In addition, the magnitude of the lower limit of the use envelope $\left(\left|N F_{\min }\right|\right)$ may be much larger than the magnitude of the upper limit of the use envelope $\left(\left|N F_{\max }\right|\right)$. Therefore, it is important in this situation to consider the model weight during the selection of the balance as it will be a large part of the total normal force that the balance will experience.

\section{Floor-Mounted Semi-Span Model}

Wind tunnel test situations exist when a strain-gage balance directly measures aerodynamic loads that act on a model. One example is the test of a semi-span model that is attached to a floor-mounted fivecomponent semi-span balance (Fig. 4a shows an example of this model configuration). A five-component semi-span balance measures the normal force, axial force, pitching moment, yawing moment, and rolling moment. The weight of the model acts, by design, in the direction of the pitch axis of the balance. The pitch axis is parallel to the gravitational acceleration. Therefore, the weight has no influence on the gage outputs of the semi-span balance. Figure 5 shows the normal force of the floor-mounted semi-span balance plotted versus the angle of attack of the semi-span model. The weight makes no contribution to the normal force that the balance measures during an angle of attack sweep.

\section{E. Rotated Full-Span Model}

Sometimes, it is possible to rotate a full-span model $90^{\circ}$ around the tunnel centerline and use the support system's original yaw angle range for an angle of attack sweep (Fig. 4b shows an example of this model configuration). In that case, assuming that the rotated model is not yawed, the gravitational acceleration is parallel to the direction of the pitch axis of the balance. Consequently, the rotated model's weight no longer influences the normal and axial force measurements. In other words - the normal force characteristics of the rotated balance will be similar to those of the floor-mounted semi-span balance (see Fig. 5).

\section{F. Relative Error of the Lift Force Measurement}

Differences in the accuracy of the measurement of the lift force for the first two wind tunnel test cases above can more easily be understood if the relative error of the lift force measurement is investigated in 
detail. In principle, this error can be expressed as follows

$$
\Lambda=\frac{\Delta L}{L_{\max }} \cdot 100 \%
$$

where $\Delta L$ is a fixed error that is associated with balance design and calibration analysis characteristics and $L_{\max }$ is the maximum lift force that is expected during the wind tunnel test. We also know that the error in the lift force measurement approximately equals the error in the normal force measurement for tests conducted within the limits of the linear lift slope range of a typical wind tunnel model. Therefore, we get:

$$
\Delta L \approx \Delta N F
$$

Then, after using the right-hand side of Eq. (10) to replace $\Delta L$ in Eq. (9), we get:

$$
\Lambda \approx \frac{\Delta N F}{L_{\max }} \cdot 100 \%
$$

In addition, assuming that a force balance is used for a wind tunnel test, we know from Ref. [3] that

$$
N F=N 1+N 2
$$

Therefore, a conservative estimate of the normal force error is given by the following inequality:

$$
\Delta N F \leq \Delta N 1+\Delta N 2
$$

Now, after applying the inequality (13) to Eq. (11), we get the relationship:

$$
\Lambda \leq \frac{\Delta N 1+\Delta N 2}{L_{\max }} \cdot 100 \%
$$

Force balances are typically designed such that the capacity of the forward normal force gage matches the capacity of the aft normal force gage. Therefore, both gage sensitivities and related absolute errors of the forward and aft force measurements are more or less identical. This conclusion can be expressed as follows:

$$
\Delta N 1 \approx \Delta N 2
$$

Consequently, after applying the approximation given in Eq. (15), inequality (14) becomes:

$$
\Lambda \leq \frac{2 \cdot \Delta N 1}{L_{\max }} \cdot 100 \%
$$

Repeat data points can be taken during a balance calibration by loading and unloading the balance during a load series. They are a good indicator of the combined load prediction error that is associated with (i) output measurement inaccuracies and (ii) loading process imperfections. Observed small changes of the electrical outputs of these types of repeats can be used in combination with the sensitivity of the balance gage in order to get an estimate of the absolute error of the forward and aft normal force measurements. It is the author's experience that outputs of very good repeat data show a maximum variation of $0.5[\mu \mathrm{V} / \mathrm{V}]$. This observation can be summarized as follows for the output of the forward normal force gage:

$$
\text { observed output change of repeat points } \Longrightarrow \Delta r N 1 \leq 0.5[\mu V / V]
$$

The sensitivity of the forward normal force gage is defined as a partial derivative. We get:

$$
S(r N 1)=\frac{\partial r N 1}{\partial N 1} \approx \frac{\Delta r N 1}{\Delta N 1}
$$

Then, after rearranging terms in Eq. (18a), we get:

$$
\Delta N 1 \approx \frac{\Delta r N 1}{S(r N 1)}
$$


In the next step, after applying the inequality (17) to Eq. (18b), we get the conservative estimate:

$$
\Delta N 1 \leq \frac{0.5[\mu V / V]}{S(r N 1)}
$$

Finally, after combining inequality (16) with inequality (19), we get the following conservative estimate of the relative error of the lift force measurement:

\section{RELATIVE ERROR OF THE LIFT FORCE MEASUREMENT}

$$
\Lambda \leq \underbrace{\frac{1.0[\mu \mathrm{V} / \mathrm{V}]}{S(r N 1)}}_{\text {balance specific }} \cdot \underbrace{\frac{1}{L_{\max }}}_{\text {use envelope }} \cdot 100 \%
$$

Inequality (20) above clearly shows how (i) a balance design characteristic like the sensitivity of the normal force gages and (ii) a use envelope characteristic like the maximum lift force influence the relative error of the lift force measurement. An increase of the relative error of the lift force measurement is usually directly associated with a decrease of the lift force measurement itself (compare, e.g., Fig. 3a with Fig. 3b). However, this unwanted increase of the relative error could be reduced by selecting a balance with a higher normal force sensitivity that better matches the expected normal force range during the wind tunnel test.

A quantitative description of the expected use envelope of the normal force of a strain-gage balance during a wind tunnel test is developed in the next section of the paper.

\section{Use Envelope Description}

In general, it is useful to have a quantitative description of the use envelope of the normal force of a strain-gage balance so that a customer has a good understanding how (i) aerodynamic characteristics of the model (lift coefficient), (ii) model size (reference area), (iii) model weight, and (iv) dynamic pressure influence the normal force range during a test. A description of the use envelope can be obtained by going back to the approximation of the connection of the three principle forces that is given in Eq. (3). We get the following alternate representation of Eq. (3) after dividing both sides of the equation by the model weight:

$$
\frac{N F}{W} \approx-1+\frac{L}{W}
$$

The right-hand side of Eq. (21) indicates that $L / W$, i.e., the "lift-to-weight ratio" of the wind tunnel model, is an important parameter that helps simplify the description of the use envelope of the normal force of the balance. Therefore, it is useful to analyze this parameter in more detail. The lift force is related to lift coefficient $\left(c_{L}\right)$, dynamic pressure $(q)$, and model reference area $(A)$ by the following relationship:

$$
L=c_{L} \cdot q \cdot A
$$

Then, after replacing the lift by the right-hand side of Eq. (22), the "lift-to-weight" ratio becomes:

$$
\frac{L}{W}=\frac{c_{L} \cdot q \cdot A}{W}
$$

The dynamic pressure in compressible flow depends on the "tunnel conditions" that are characterized by the total pressure $\left(p_{T}\right)$ and the Mach number $(M)$. The connection between dynamic pressure and tunnel conditions is given by the following equation (taken from Ref. [4]):

$$
q=p_{T} \cdot \frac{\gamma}{2} \cdot M^{2} \cdot\left[1+\frac{\gamma-1}{2} \cdot M^{2}\right]^{\frac{-\gamma}{\gamma-1}}
$$


Then, knowing that the ratio of specific heats $(\gamma)$ of air equals $\approx 1.4$, Eq. $(24)$ can be expressed as follows:

$$
\text { air } \Longrightarrow q=p_{T} \cdot \frac{7}{10} \cdot M^{2} \cdot\left[1+\frac{M^{2}}{5}\right]^{-7 / 2}
$$

It is useful for the definition of the lift-to-weight ratio of the model to make the dynamic pressure dimensionless. This goal can easily be achieved after dividing both sides of Eq. (25) by the mean sea-level pressure $\left(p_{T \circ}\right)$. Then, the dimensionless dynamic pressure $(\delta)$ can be described by the following relationship:

\section{DIMENSIONLESS DYNAMIC PRESSURE FOR AIR}

$$
\begin{aligned}
\delta=\frac{q}{p_{T \circ}}= & \underbrace{\left[\frac{p_{T}}{p_{T \circ}}\right]}_{\text {pressure ratio }} \cdot \frac{7}{10} \cdot M^{2} \cdot\left[1+\frac{M^{2}}{5}\right]^{-7 / 2} \\
\text { where } & \\
p_{T \circ} & =2116.2\left[\mathrm{lbf} / \mathrm{ft}^{2}\right] \text { or } 101325\left[\mathrm{~N} / \mathrm{m}^{2}\right]
\end{aligned}
$$

We know from Eq. (26a) that the dynamic pressure can be expressed as the product of dimensionless dynamic pressure and mean sea-level pressure. Then, we get:

$$
q=\delta \cdot p_{T \circ}
$$

Now, the right-hand side of Eq. (27) can be used to replace the dynamic pressure in Eq. (23) that defines the lift-to-weight ratio. Then, after rearranging terms, the lift-to-weight ratio becomes:

$$
\frac{L}{W}=c_{L} \cdot \delta \cdot \frac{p_{T \circ} \cdot A}{W}
$$

The left-hand side of Eq. (28) is dimensionless. The parameters $c_{L}$ and $\delta$ on the right-hand side of Eq. (28) are also dimensionless. Consequently, the remaining fraction on the right-hand side of Eq. (28) must also be a dimensionless quantity. In fact, the fraction can be interpreted as the dimensionless weight of the wind tunnel model. Then, after rearranging the fraction, we can write:

\section{DIMENSIONLESS MODEL WEIGHT}

$$
\omega=\frac{1}{p_{T \circ}} \cdot \frac{W}{A}
$$

The introduction of the dimensionless model weight has several advantages. First, models of different size can directly be compared with each other. In addition, the definition of the dimensionless model weight makes it possible to use terms like "light" or "heavy" for the description of a model as the metric relates the "raw" model weight to the model's reference area. Finally, after replacing the fraction on the right-hand side of Eq. (28) with the inverse of the dimensionless model weight, the model's lift-to-weight ratio becomes:

\section{LIFT-TO-WEIGHT RATIO OF A MODEL}

$$
\frac{L}{W}=\frac{c_{L} \cdot \delta}{\omega}
$$


The right-hand side of Eq. (30) shows that the lift-to-weight ratio of the wind tunnel model can be expressed as a function of three dimensionless quantities $\left(c_{L}, \delta, \omega\right)$. This description also makes it obvious how the lift-to-weight ratio of the model can be influenced in order to increase the use envelope of the normal force of the balance. In general, it is desirable to maximize the lift-to-weight ratio of the model. The lift coefficient $\left(c_{L}\right)$ is a function of the aerodynamic characteristics of the test article. It cannot be influenced because it is the ultimate goal of the lift force measurement. However, a simple increase of the total pressure $\left(p_{T}\right)$ will increase the dimensionless dynamic pressure $(\delta)$ and, consequently, increase the model's lift-toweight ratio. Similarly, the lift-to-weight ratio could also be increased by reducing the dimensionless model weight $(\omega)$. This reduction can be accomplished by minimizing the ratio between model weight and model reference area $(W / A)$ while still meeting the model's stress limits during the test.

It remains to connect the lift-to-weight ratio to the normal force range so that the lower and upper bound of the use envelope of the normal force can be defined within the limits of the linear lift slope range. The bounds of the model's lift coefficient for the linear lift slope range can be described by the following relationship:

$$
c_{\text {Lmin }} \leq c_{L} \leq c_{\text {Lmax }}
$$

Consequently, keeping in mind that the lift-to-weight ratio has either a positive or negative sign depending on the sign of the lift coefficient, we get the following range for the lift-to-weight ratio:

$$
\frac{L_{\min }}{W} \leq \frac{L}{W} \leq \frac{L_{\max }}{W}
$$

where the lower and upper bounds can also be expressed as

$$
\begin{aligned}
\text { lower bound } \Longrightarrow \frac{L_{\min }}{W} & =\frac{c_{L \min } \cdot \delta}{\omega} \\
\text { upper bound } \Longrightarrow \frac{L_{\max }}{W} & =\frac{c_{\text {Lmax }} \cdot \delta}{\omega}
\end{aligned}
$$

Finally, after combining the definition of the lower and upper bound of the model's lift-to-weight ratio with the description of the normal force of the balance that is given in Eq. (21), we get the desired quantitative description of the use envelope of the normal force of the balance:

\section{USE ENVELOPE OF THE NORMAL FORCE}

$$
\begin{aligned}
& \frac{N F_{\min }}{W} \leq \frac{N F}{W} \leq \frac{N F_{\max }}{W} \\
& \text { where } \\
& \frac{N F_{\min }}{W}=-1+\frac{c_{\text {Lmin }} \cdot \delta}{\omega} \\
& \frac{N F_{\max }}{W}=-1+\frac{c_{L \max } \cdot \delta}{\omega}
\end{aligned}
$$

It will be illustrated in the next section of the paper how the quantitative description of the use envelope of the normal force can be applied to real-world wind tunnel test situations. Typical data from tests in the Ames 11-Ft Transonic Wind Tunnel will be used for this purpose.

\section{Discussion of Example}

In the previous section it was shown that the lift-to-weight ratio of the wind tunnel model links the aerodynamic characteristics of the model $\left(c_{L}\right)$, the tunnel conditions $(\delta)$, and the physical model size $(\omega)$ to the use envelope of the normal force of the balance during a wind tunnel test. The maximum lift coefficient for

American Institute of Aeronautics and Astronautics 
models tested in transonic flow is often near one (see, e.g., results reported in Ref. [5] for NASA's Common Research Model). After applying this simplification to Eq. (33c), we get for the upper bound of the use envelope the following relationship:

$$
\underbrace{c_{\text {Lmax }} \approx 1.0}_{\text {model in transonic flow }} \Longrightarrow \frac{N F_{\max }}{W}=-1+\frac{\delta}{\omega}
$$

The Ames 11-Ft Transonic Wind Tunnel is a closed-circuit tunnel that can vary the total pressure from one half to two atmospheres. The Mach number ranges from 0.2 to 1.4. The author decided to only consider subsonic Mach numbers during the discussion of the example. The calculation of the lift-to-weight ratio of the model requires estimates of the dimensionless dynamic pressure. Table 1 lists these values for various combinations of Mach number and total pressure ratio.

Table 1: Dimensionless dynamic pressure $\delta$ for typical total pressure ratios and Mach numbers.

\begin{tabular}{|r|c|c|c|c|c|c|c|c|c|c|}
\hline$M \Longrightarrow$ & 0.20 & 0.30 & 0.40 & 0.50 & 0.60 & 0.70 & 0.80 & 0.85 & 0.90 & 0.95 \\
\hline \hline$p_{T} / p_{T \circ}=0.5$ & 0.014 & 0.030 & 0.050 & 0.074 & 0.099 & 0.124 & 0.147 & 0.158 & 0.168 & 0.177 \\
$p_{T} / p_{T \circ}=1.0$ & 0.027 & 0.059 & 0.100 & 0.148 & 0.198 & 0.247 & 0.294 & 0.315 & 0.335 & 0.353 \\
$p_{T} / p_{T \circ}=2.0$ & 0.054 & 0.118 & 0.201 & 0.295 & 0.395 & 0.495 & 0.588 & 0.631 & 0.670 & 0.707 \\
\hline
\end{tabular}

The dimensionless model weight is also needed for the calculation of the lift-to-weight ratio. This parameter depends on the type and size of wind tunnel model that is tested. Data from past wind tunnel tests of airplane models indicates that the dimensionless model weight of typical models tested in the Ames 11-Ft Transonic Wind Tunnel has the following range:

$$
\omega \approx 0.025 \ldots 0.050
$$

Data from the 2010 test of NASA's Common Research Model in the Ames 11-Ft Transonic Wind Tunnel can be used to illustrate the calculation of the dimensionless model weight. During this test the absolute weight of the model was precisely determined by leveling the model in the tunnel, recording the electrical outputs of the balance gages in "wind-off" condition, and, afterwards, processing these outputs using the load prediction matrix of the balance that was installed in the model to measure model loads. These calculations resulted in an estimated model weight (as installed in the tunnel) of $306[l b f]$ (or $1361[N]$ ). The model's reference area was given as $3.01\left[f t^{2}\right]$ (or $0.2796\left[\mathrm{~m}^{2}\right]$ ). Then, after applying Eq. (29), we get for the dimensionless model weight of the Common Research Model the following result:

$$
\omega=\frac{306[l b f]}{2116.2\left[l b f / f t^{2}\right] \times 3.01\left[f t^{2}\right]}=\frac{1361[N]}{101325\left[\mathrm{~N} / \mathrm{m}^{2}\right] \times 0.2796\left[\mathrm{~m}^{2}\right]} \approx 0.048
$$

Now, all information is available to compute the model's lift-to-weight ratio for the different Mach number and total pressure combinations that are listed in Table 1. First, the lift-to-weight ratio is computed for a "light" model that is at the lower end of the tunnel's dimensionless model weight range. Table 2 lists corresponding values of the lift-to-weight ratio assuming that the maximum lift coefficient is one.

Table 2: Lift-to-weight ratio table for a "light" wind tunnel model with $\omega=0.025$ and $c_{\text {Lmax }}=1$.

\begin{tabular}{|r|c|c|c|c|c|c|c|c|c|c|}
\hline$M \Longrightarrow$ & 0.20 & 0.30 & 0.40 & 0.50 & 0.60 & 0.70 & 0.80 & 0.85 & 0.90 & 0.95 \\
\hline \hline$p_{T} / p_{T \circ}=0.5$ & $<1$ & 1.2 & 2.0 & 3.0 & 4.0 & 4.9 & 5.9 & 6.3 & 6.7 & 7.1 \\
$p_{T} / p_{T \circ}=1.0$ & 1.1 & 2.4 & 4.0 & 5.9 & 7.9 & 9.9 & $>10$ & $>10$ & $>10$ & $>10$ \\
$p_{T} / p_{T \circ}=2.0$ & 2.2 & 4.7 & 8.0 & $>10$ & $>10$ & $>10$ & $>10$ & $>10$ & $>10$ & $>10$ \\
\hline
\end{tabular}

Lift-to-weight ratios listed in Table 2 show expected trends: the ratio increases with both increasing Mach number and increasing total pressure ratio. The benefit of testing at a higher total pressure ratio also 
becomes obvious. A doubling of the total pressure ratio, for example, doubles the lift-to-weight ratio of the model. In the next step, the lift-to-weight ratio is computed for a "heavy" model that is at the upper end of the tunnel's dimensionless model weight range. Table 3 lists corresponding values of the lift-to-weight ratio for this model configuration assuming again that the maximum lift coefficient is one.

Table 3: Lift-to-weight ratio table for a "heavy" wind tunnel model with $\omega=0.050$ and $c_{\text {Lmax }}=1$.

\begin{tabular}{|c|c|c|c|c|c|c|c|c|c|c|}
\hline$M \Longrightarrow$ & 0.20 & 0.30 & 0.40 & 0.50 & 0.60 & 0.70 & 0.80 & 0.85 & 0.90 & 0.95 \\
\hline \hline$p_{T} / p_{T \circ}=0.5$ & $<1$ & $<1$ & 1.0 & 1.5 & 2.0 & 2.5 & 2.9 & 3.2 & 3.4 & 3.5 \\
$p_{T} / p_{T \circ}=1.0$ & $<1$ & 1.2 & 2.0 & 3.0 & 4.0 & 4.9 & 5.9 & 6.3 & 6.7 & 7.1 \\
$p_{T} / p_{T \circ}=2.0$ & 1.1 & 2.4 & 4.0 & 5.9 & 7.9 & 9.9 & $>10$ & $>10$ & $>10$ & $>10$ \\
\hline
\end{tabular}

As expected, lift-to-weight ratios listed in Table 3 show the same trends as the ratios listed in Table 2. However, it is observed that the increase of the dimensionless model weight from 0.025 to 0.050 noticeably reduces the lift-to-weight ratios.

It is possible to develop a universally applicable lift-to-weight ratio table that can be used in any subsonic wind tunnel. First, it is assumed that both the lift coefficient and the total pressure ratio equal one. Then, using a reasonable set of discrete Mach numbers and dimensionless model weights, the liftto-weight ratio is computed for all possible combinations of these two parameters. An example of such a universally applicable lift-too-weight ratio table is given in Table 4 below.

Table 4: Universally applicable lift-to-weight ratio table for $c_{L}=1$ and $p_{T} / p_{T \circ}=1$.

\begin{tabular}{|c|c|c|c|c|c|c|c|c|c|c|}
\hline$M \Longrightarrow$ & 0.20 & 0.30 & 0.40 & 0.50 & 0.60 & 0.70 & 0.80 & 0.85 & 0.90 & 0.95 \\
\hline \hline$\omega=0.010$ & 2.7 & 5.9 & 10.0 & $>10$ & $>10$ & $>10$ & $>10$ & $>10$ & $>10$ & $>10$ \\
$\omega=0.015$ & 1.8 & 3.9 & 6.7 & 9.8 & $>10$ & $>10$ & $>10$ & $>10$ & $>10$ & $>10$ \\
$\omega=0.020$ & 1.4 & 3.0 & 5.0 & 7.4 & 9.9 & $>10$ & $>10$ & $>10$ & $>10$ & $>10$ \\
$\omega=0.025$ & 1.1 & 2.4 & 4.0 & 5.9 & 7.9 & 9.9 & $>10$ & $>10$ & $>10$ & $>10$ \\
$\omega=0.030$ & $<1$ & 2.0 & 3.3 & 4.9 & 6.6 & 8.2 & 9.8 & $>10$ & $>10$ & $>10$ \\
$\omega=0.035$ & $<1$ & 1.7 & 2.9 & 4.2 & 5.6 & 7.1 & 8.4 & 9.0 & 9.6 & $>10$ \\
$\omega=0.040$ & $<1$ & 1.5 & 2.5 & 3.7 & 4.9 & 6.2 & 7.3 & 7.9 & 8.4 & 8.8 \\
$\omega=0.045$ & $<1$ & 1.3 & 2.2 & 3.3 & 4.4 & 5.5 & 6.5 & 7.0 & 7.4 & 7.9 \\
$\omega=0.050$ & $<1$ & 1.2 & 2.0 & 3.0 & 4.0 & 4.9 & 5.9 & 6.3 & 6.7 & 7.1 \\
$\omega=0.055$ & $<1$ & 1.1 & 1.8 & 2.7 & 3.6 & 4.5 & 5.3 & 5.7 & 6.1 & 6.4 \\
$\omega=0.060$ & $<1$ & 1.0 & 1.7 & 2.5 & 3.3 & 4.1 & 4.9 & 5.3 & 5.6 & 5.9 \\
\hline
\end{tabular}

Lift-to-weight ratios listed in Table 4 can easily be scaled to alternate lift coefficients and total pressure ratios by taking advantage of the direct proportionality between the lift-to-weight ratio and these two parameters. For example, we know from Eq. (30) that the following two proportionalities are valid

$$
\begin{aligned}
& \frac{L}{W} \propto c_{L} \\
& \frac{L}{W} \propto \delta
\end{aligned}
$$

In addition, we know from Eq. (26a) that the following proportionality applies:

$$
\delta \propto \frac{p_{T}}{p_{T \circ}}
$$

Then, after applying Eq. (38) to Eq. (37b), it is concluded that the following proportionality applies: 


$$
\frac{L}{W} \propto \frac{p_{T}}{p_{T \circ}}
$$

Consequently, we get the following general equation that expresses the lift-to-weight ratio of the wind tunnel model as a function of the lift coefficient and the total pressure ratio:

$$
\frac{L}{W}\left(c_{L} ; \frac{p_{T}}{p_{T \circ}}\right)=\underbrace{\frac{L}{W}\left(c_{L}=1 ; \frac{p_{T}}{p_{T \circ}}=1\right)}_{\text {input from Table } 4} \cdot c_{L} \cdot \frac{p_{T}}{p_{T \circ}}
$$

Values listed in Table 4 in combination with the scaling defined in Eq. (40) allow a wind tunnel user to get a rapid estimate of the lift-to-weight ratio of the wind tunnel model so that a better understanding of the expected absolute accuracy of the lift force measurements can be obtained.

\section{Summary and Conclusions}

The influence of the wind tunnel model's weight on the use envelope of the normal force of a strain-gage balance during a wind tunnel test was discussed. First, the fundamental relationship between normal force, model size, model weight, dynamic pressure, angle of attack, and lift coefficient was derived. Then, four typical wind tunnel test cases were compared that illustrate different aspects of the influence of the model weight on the lift force measurement. The relative error of the lift force measurement was introduced in order to connect the normal force resolution capability of the balance to the maximum lift force that the model experiences during a test. In addition, the lift-to-weight ratio of the wind tunnel model was defined as a function of three dimensionless quantities (lift coefficient, dimensionless dynamic pressure, dimensionless model weight) so that a quantitative description of the use envelope of the normal force could be developed.

The current study clearly shows that low speed tests of "heavy" wind tunnel models, i.e., models with a large dimensionless model weight, present the greatest challenge to an accurate measurement of the lift force. In that case, the model weight could significantly reduce the use envelope of the normal force of the balance, and, consequently, increase the relative error associated with the lift force measurements.

Several actions can be taken to improve the overall accuracy of the lift force measurements during low speed tests. For example, the negative influence of a large dimensionless model weight can be reduced by increasing the lift-to-weight ratio of the wind tunnel model. This increase is accomplished by increasing the total pressure during the wind tunnel test. Then, the use envelope of the normal force of the balance is increased and, consequently, the relative error associated with the lift force measurement is reduced. A total pressure increase, of course, has a second unrelated benefit: the wind tunnel test is conducted closer to the model's expected full-scale Reynolds Number.

An increase of the model's lift-to-weight ratio can also be achieved by redesigning the model such that the dimensionless model weight is minimized while still meeting expected stress limits on the model. A change of the lift-to-weight ratio of the model is often not possible. Then, a reduction of the negative influence of the model weight on the accuracy of the lift force measurement can still be achieved by selecting an alternate balance for the low speed test that has a greater normal force sensitivity. It may also be possible for some full-span model configurations to simply rotate the test article $90^{\circ}$ around the tunnel centerline and use the support system's original yaw angle range for the desired angle of attack sweep. Then, the model weight has no influence on both the normal and axial force measurements because the gravitational acceleration is perpendicular to the plane that is spanned by the normal and axial force of the balance.

Improvements in force measurement accuracy can become expensive because all suggested actions (test at higher total pressures, redesign of the wind tunnel model, selection of an alternate balance with higher normal force sensitivity, model rotation) will most likely increase cost and/or limit the scope of the wind 
tunnel test. Therefore, a wind tunnel customer needs to have a good understanding of the final accuracy requirement for the lift force measurements to make best use of available resources.

\section{Acknowledgements}

The author wants to thank Bob Gisler and Ray Rhew of NASA for their critical and constructive review of the final manuscript. The work reported in this paper was supported by the Wind Tunnel Division at NASA Ames Research Center under contract NNA09DB39C.

\section{References}

${ }^{1}$ AIAA/GTTC Internal Balance Technology Working Group, Recommended Practice - Calibration and Use of Internal Strain-Gage Balances with Application to Wind Tunnel Testing, AIAA R-091-2003, American Institute of Aeronautics and Astronautics, Reston, Virginia, 2003, p. 3, Fig. 1.

${ }^{2}$ Pope, A., and Goin, K. L., High-Speed Wind Tunnel Testing, 1st edition, John Wiley \& Sons, Inc., New York, 1965, pp. 242-283, Fig. 7:1.

${ }^{3}$ Ulbrich, N., and Bader, J., "Analysis of Sting Balance Calibration Data Using Optimized Regression Models," AIAA 2009-5372, paper presented at the 45th AIAA/ASME/SAE/ASEE Joint Propulsion Conference, Denver, Colorado, August 2009, Eq. (A.1).

${ }^{4}$ Ames Research Staff, Equations, Tables, and Charts for Compressible Flow, NACA Report 1135, Ames Aeronautical Laboratory, Moffett Field, California, 1953, Eq. (48).

${ }^{5}$ Rivers, M. B., and Dittberner, A., "Experimental Investigations of the NASA Common Research Model in the NASA Langley National Transonic Facility and NASA Ames 11-Ft Transonic Wind Tunnel," AIAA 2011-1126, paper presented at the 49th AIAA Aerospace Sciences, January 2011, Orlando, Florida. 


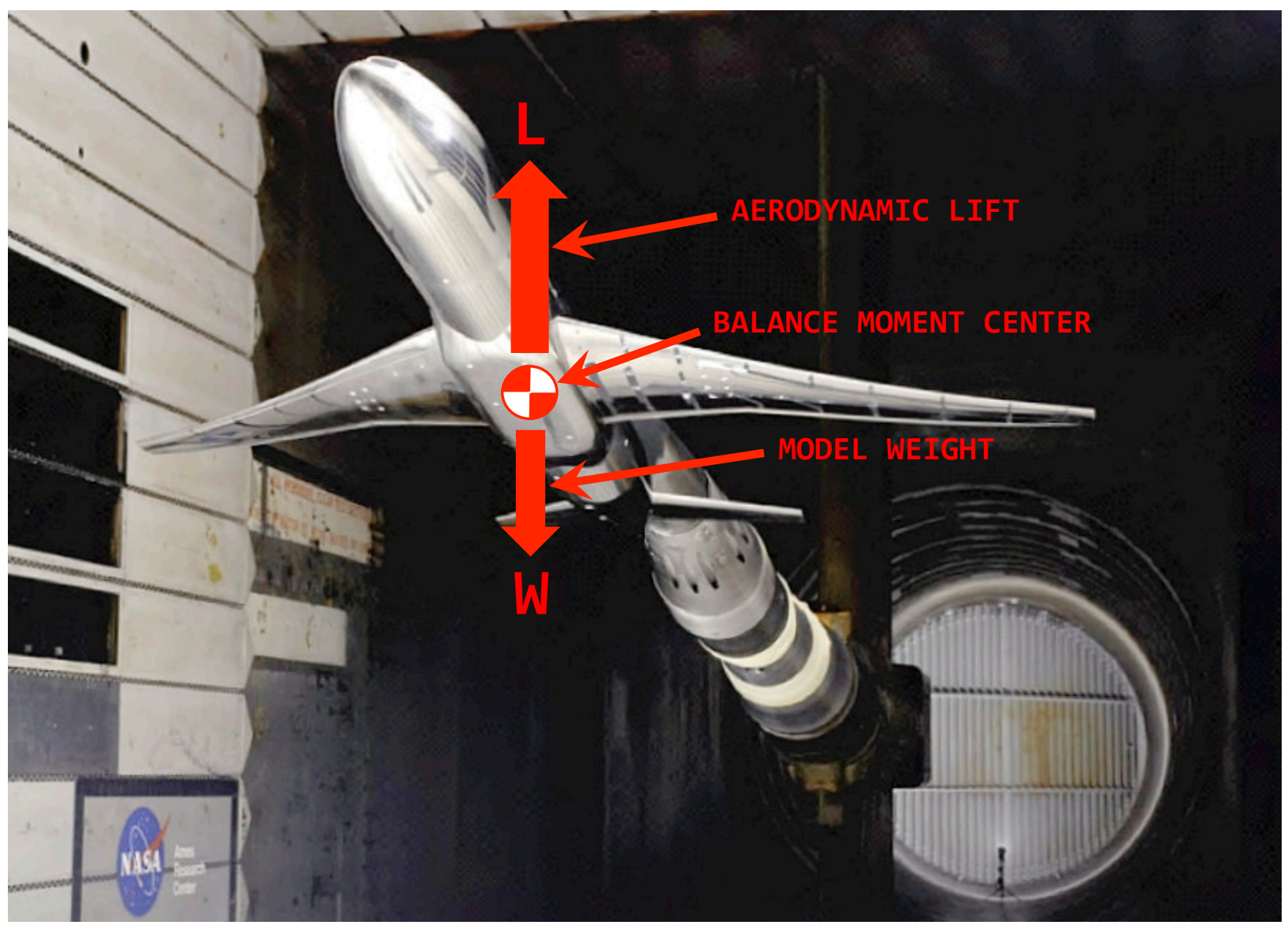

Fig. 1 Relationship between the aerodynamic lift force and the model weight during a wind tunnel test (image shows NASA's Common Research Model in the Ames 11-Ft Transonic Wind Tunnel).

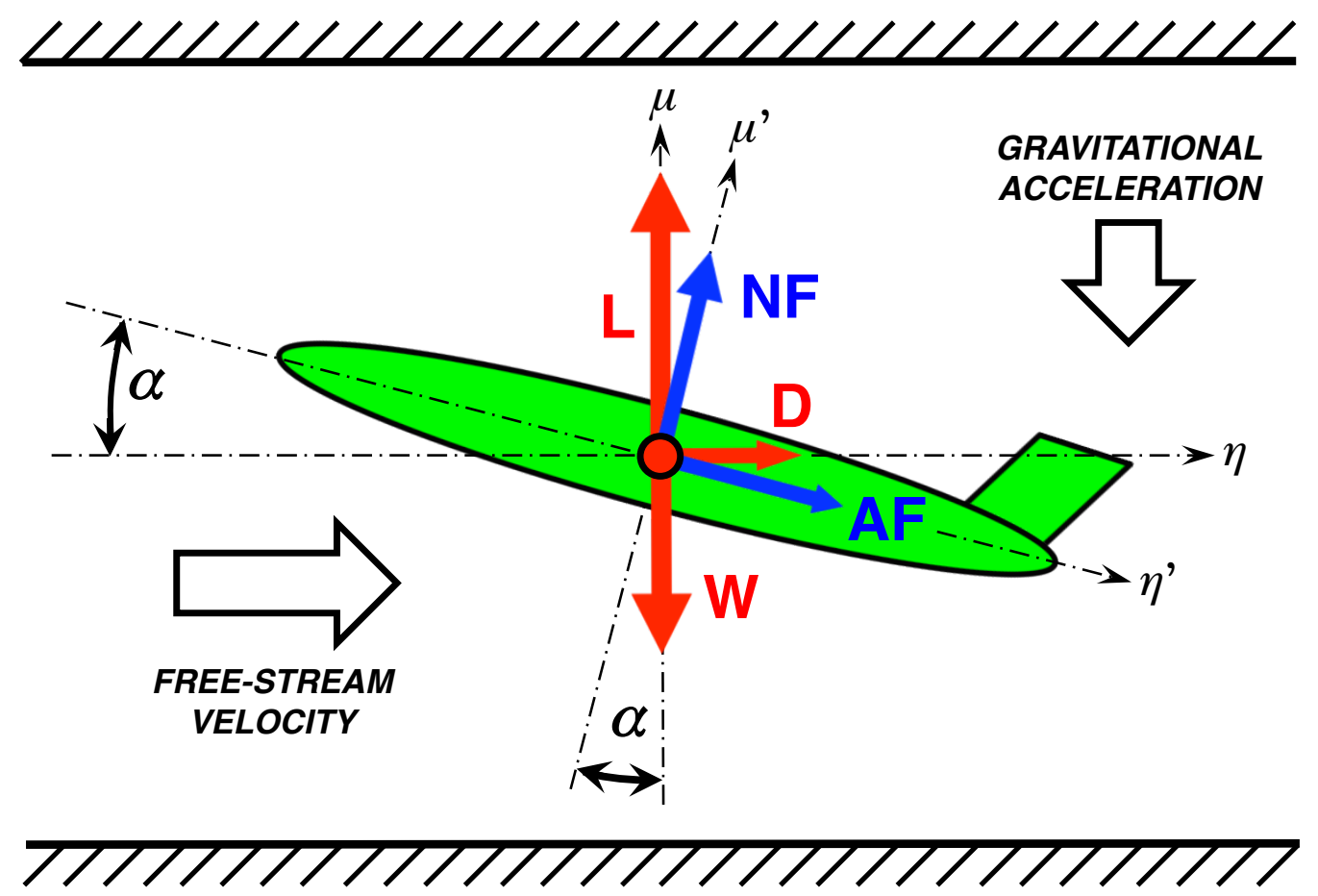

Fig. 2 Forces acting in both the wind and balance-fixed axis system of an unpowered wind tunnel model (red vector $\equiv$ force in the wind axis system ; blue vector $\equiv$ force in the balance-fixed axis system). 


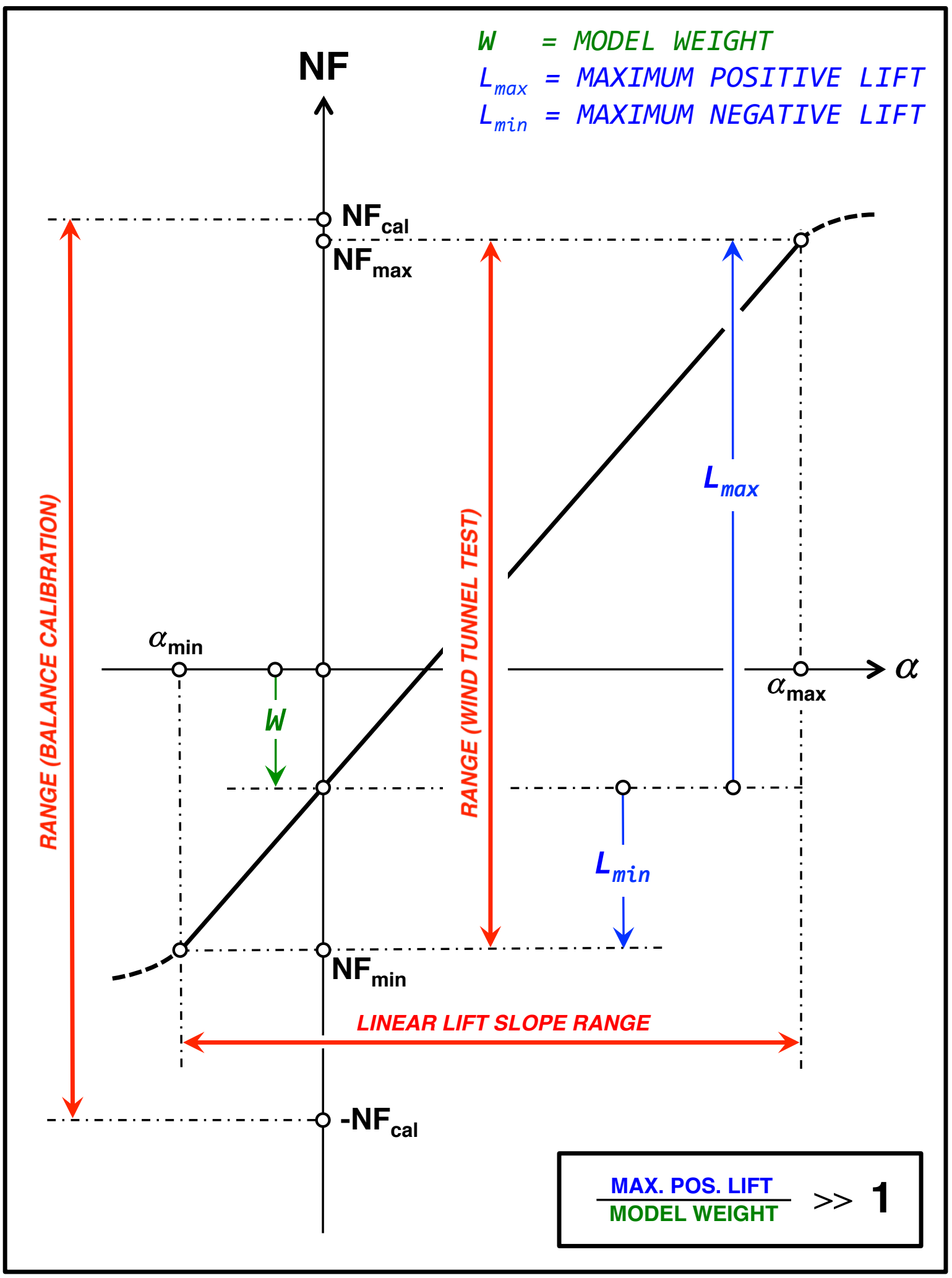

Fig. 3a Normal force characteristics for a "light" wind tunnel model tested at a high subsonic Mach number (total pressure \& Mach number are assumed to be constant during the angle of attack sweep). 


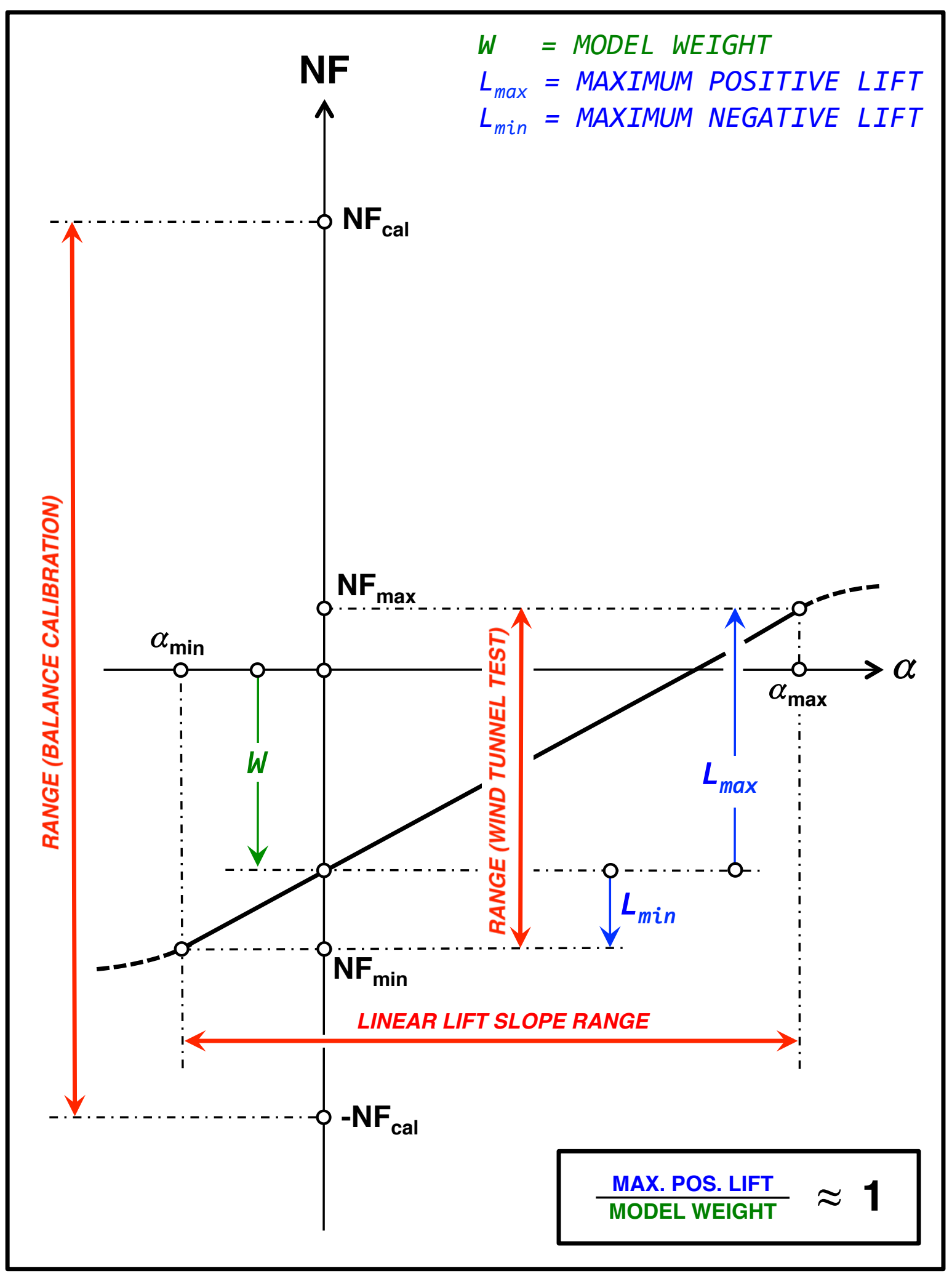

Fig. 3b Normal force characteristics for a "heavy" wind tunnel model tested at a low subsonic Mach number (total pressure \& Mach number are assumed to be constant during the angle of attack sweep). 


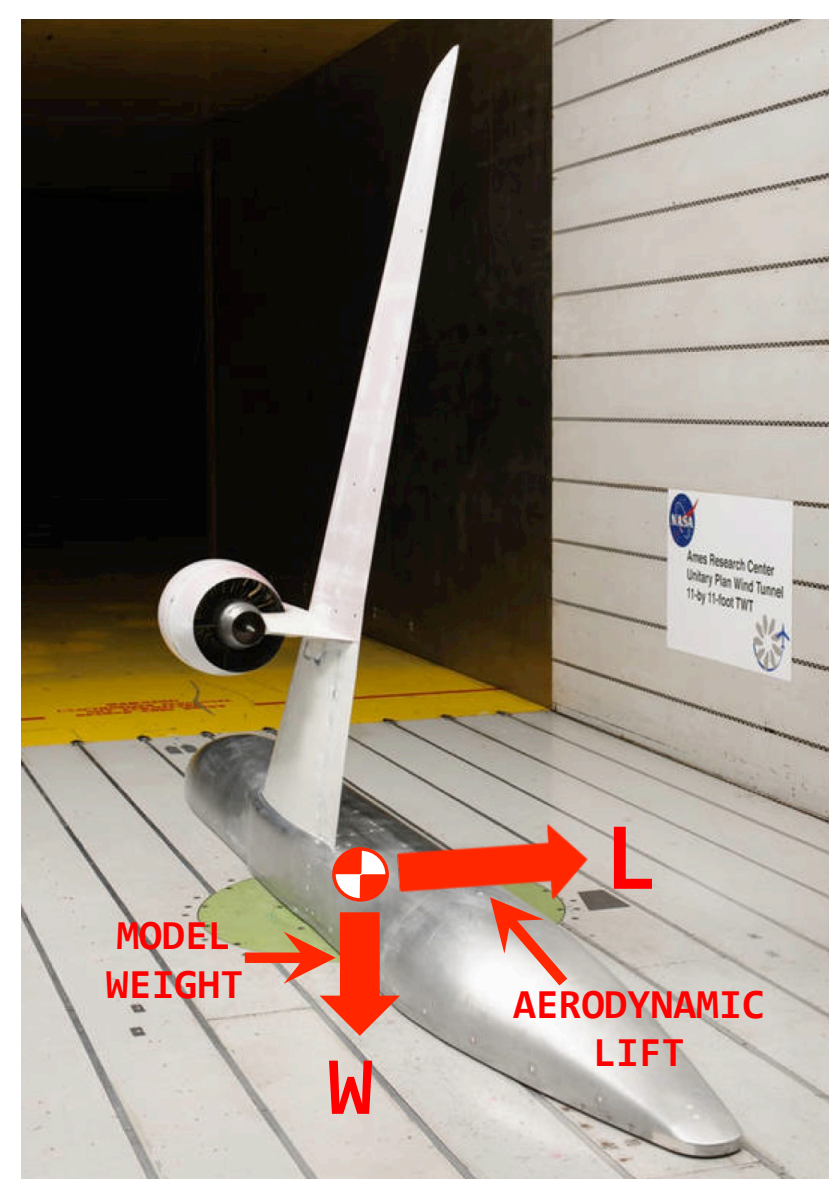

Fig. 4a Test of the Ultra-High Bypass Semi-Span Model in the NASA Ames 11-Ft Transonic Wind Tunnel (image courtesy of the Wind Tunnel Division at NASA Ames Research Center).

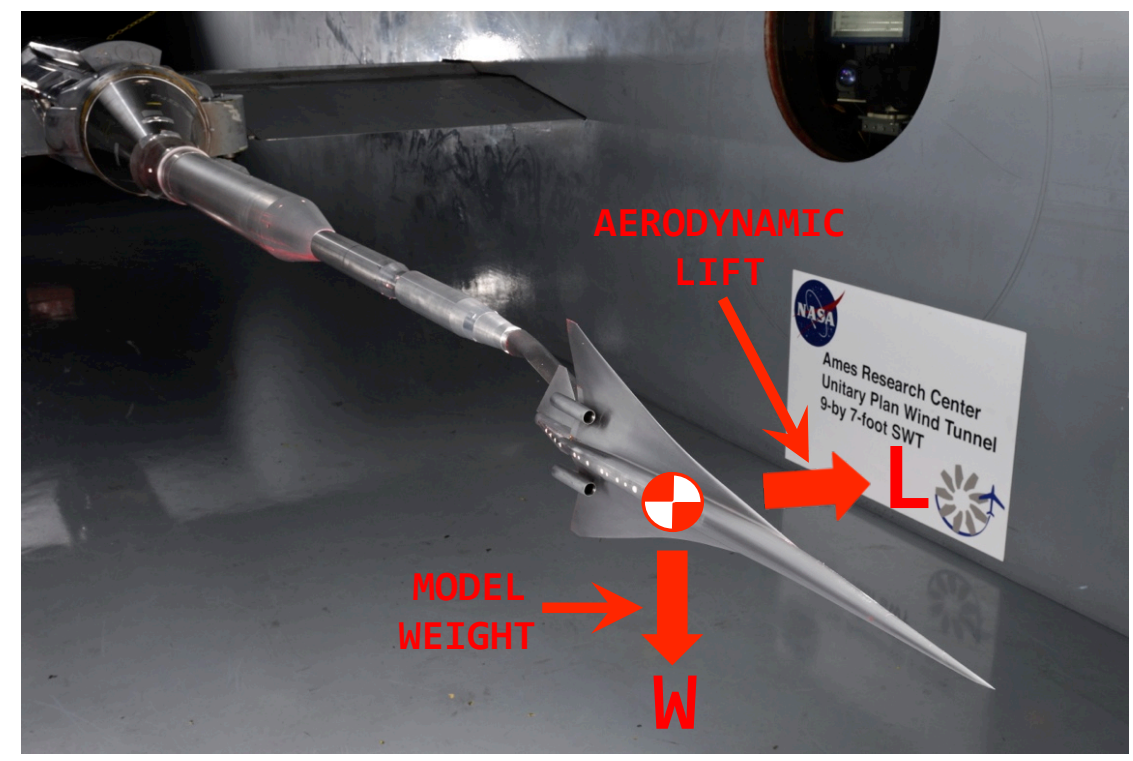

Fig. 4b Test of a sonic boom wind tunnel model in the NASA Ames $9 \times 7$ Supersonic Wind Tunnel (image courtesy of the Commercial Supersonic Technology Project at NASA Ames Research Center). 


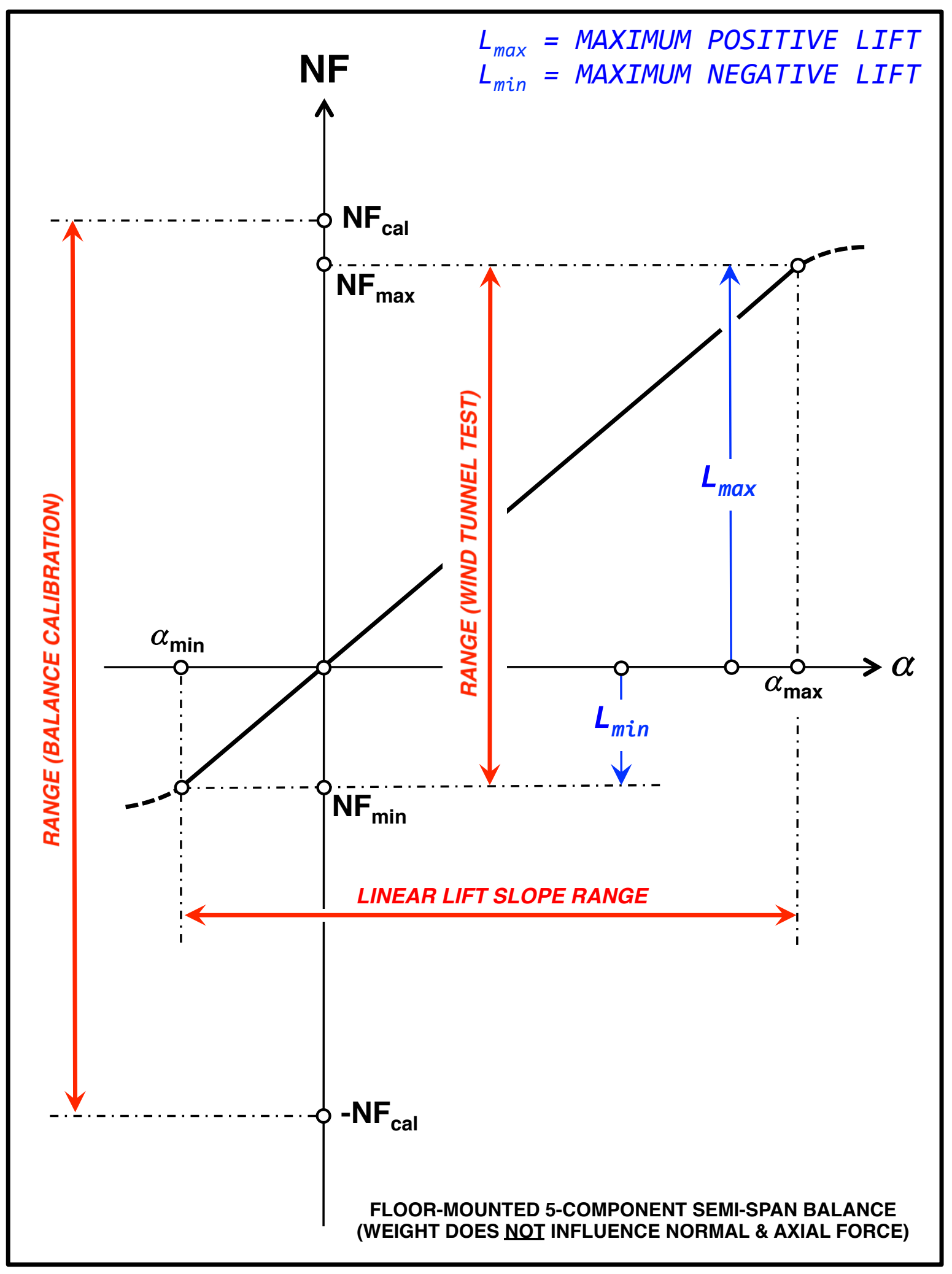

Fig. 5 Normal force characteristics for a floor-mounted semi-span model (total pressure and Mach number are assumed to be constant during the angle of attack sweep). 